\title{
COMMISSIONING AND PERFORMANCE OF LOW IMPEDANCE ELECTROSTATIC SEPARATORS FOR HIGH LUMINOSITY AT CESR
}

\author{
J.J. Welch G.W. Codner W. Lou ${ }^{\dagger}$ Cornell University, Ithaca NY
}

\section{INTRODUCTION}

Electrostatic separators have been used extensively for a number of years in colliding beam storage rings because of their ability to generate closed orbits displacements which are of opposite sign depending on the charge of the stored beams, [1], [2], [3], [4] As higher beam currents became more in demand, it was realized that separators could provide a means of multiplying the maximum beam current without needing to build a second ring to avoid unwanted collisions that additional bunches would make [5]. Much higher beam currents have resulted and have in turn placed new demands on the separator performance. To this end four new horizontal separators were designed and installed in CESR at Cornell in the fall of 1995. As of the end of 1998 they have operated with beams of up to $300 \mathrm{~mA}$ per beam. Next year they will be required to work at $500 \mathrm{~mA}$ per beam. This paper reviews their performance, especially under high current conditions.

\section{DESCRIPTION}

At CESR six electrostatic separators generate closed-orbit displacements 'pretzels', which weave back and forth around the ring. These pretzels allow the electrons and positrons to simultaneously be stored in the same vacuum chamber without destructive unwanted collisions. The lobes of the pretzel and the distribution of the bunches in the beams are carefully designed to give good separation at the numerous bunch crossing points. Four of the six separators are horizontal separators and these are the subject of this paper. The two vertical separators will not be discussed further.

The four horizontal separators are all identical ${ }^{1}$ and consist of $3 \mathrm{~m}$ long high voltage (HV) electrodes parallel to the beam. See Figure 1. Operating voltages of $\pm 85 \mathrm{kV}$ are applied to the electrodes to produce a uniform horizontal electric field of approximately $2 \mathrm{MV} / \mathrm{m}$.

The design of the separators attempted to minimize the interaction of the beam induced electromagnetic fields as well as improving the high voltage behavior. Extra 'ground electrodes' which are not needed to make the electric field, serve to confine the beam induced fields to the beam axis. These have been shown to reduce the overall loss factor by about one half. Also the ends of the vacuum chamber are

\footnotetext{
* Supported by the National Science Foundation

${ }^{\dagger}$ Formally of Cornell, now at ADC Telecommunications, CT

${ }^{1}$ One separator has an additional external resistor for faster bleed-down
}

continuously tapered and the ground electrodes smoothly joined to the ends with good rf contacts. These features gave another reduction in loss factor by about one half.

Another design issue was to limit the photoelectron current generated when synchrotron radiation (SR) strikes either the negative electrode or a part of the chamber at ground potential but with a negative electric field at the surface. Previous experience had shown that the photocurrent are usually much larger that the high voltage field emission currents, and present technical demands for the power supply and electrode cooling.

\section{PERFORMANCE}

Overall the separators have been one of most reliable systems in the accelerator. Performance issues are dominated by the effects of RF power produced by the beams and photoelectron current.

\subsection{Beam RF Resonances}

A typical bunch length in CESR is $1.8 \mathrm{~cm}$ and is much smaller than structures inside separators. So there are a multitude of possible resonances which may effectively couple to the beam. If nothing were done to damp these resonances, RF fields would rapidly build up until RF breakdown occurs, and indeed damage from such arcing has been observed [7]. Intense resonant fields have another deleterious effect in that they can degrade beam stability. Fortunately the vast majority of the resonances couple to the electrodes and the high voltage cables. In the CESR separators beam induced RF fields are propagated along the electrodes and HV cables to high power absorbers about $2 \mathrm{~m}$ from the vacuum feedthrough. away, where the RF power is dissipated as heat.

The electrodes and HV cables form a transmission line structure and will therefore propagate all frequencies as TEM waves. However, not all resonances are necessarily effectively damped. Because of various unavoidable impedance mis-matches between the cables, terminations, feedthroughs and electrode structure, standing waves can form on the high voltage cable. If a standing wave happens to have a current node at the location of the high power assembly, then the absorber would be ineffective and a high RF field level can result. This situation has occurred on at least two times at CESR. In the first occasion high RF currents built up and melted the connector between the HV cable and the separator. In the second case high RF voltage 


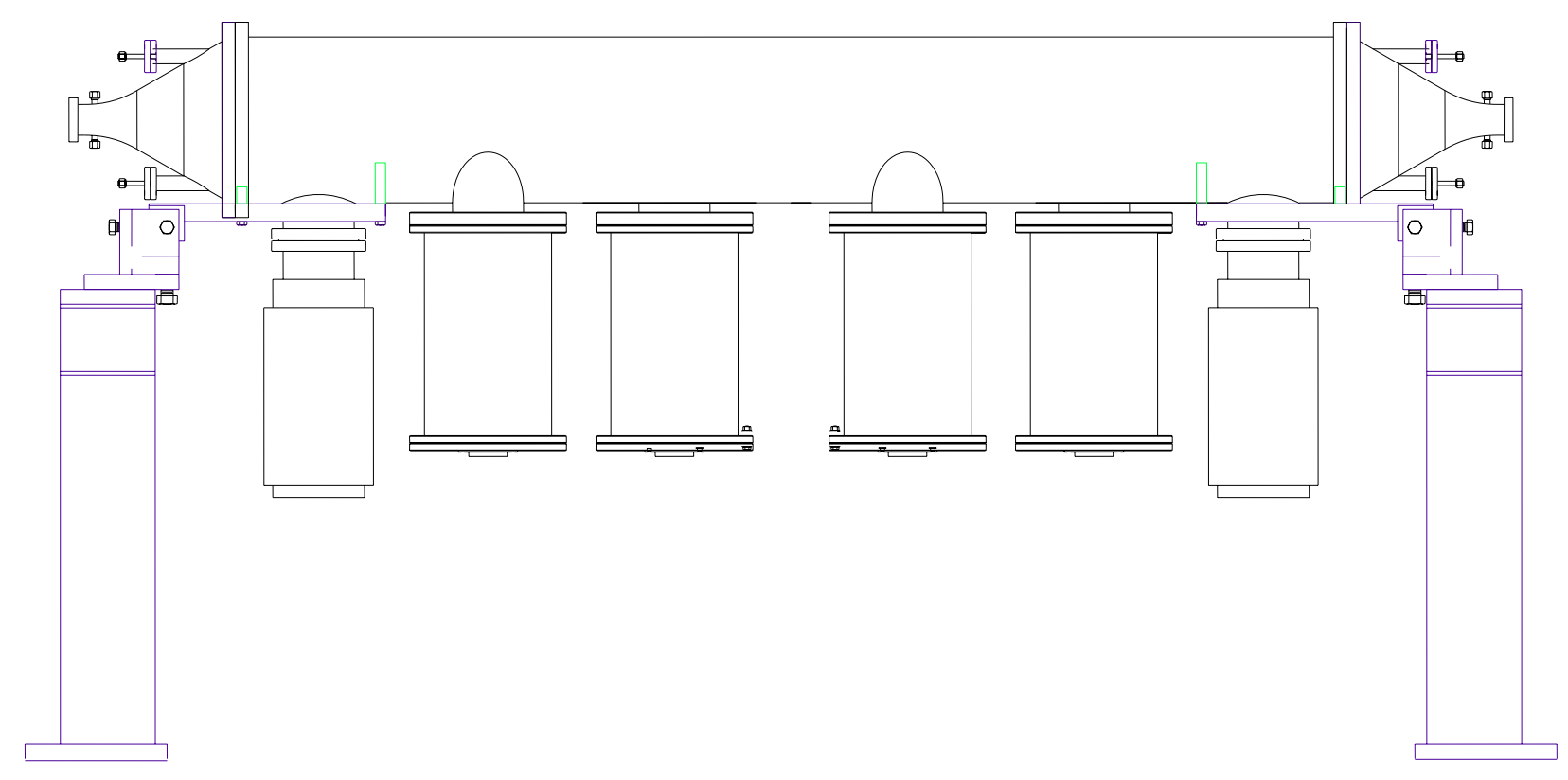

Figure 1: Low impedance electrostatic separator at CESR.

built up and caused repeated failures (HV tracking) across the $\mathrm{HV}$ connector about $3 \mathrm{~m}$ from the separator. In both cases, the problem was eliminated by changing the length of the cables, basically by trial and error, until one was found which didn't have the unfortunate phase at the absorber assembly.

\subsection{Loss Factor Measurement}

The "loss factor" is defined as the average electromagnetic energy (mainly RF) left behind by a bunch divided by the bunch charge squared. It is measured using calorimetry. The RF absorbers are cooled by essentially boiling in a bath of liquid freon (TF-113). Freon is also circulated in a closed loop to cool the electrodes and feedthroughs, and the heat is removed via a heat exchanger. The difference between the input and output temperature at the heat exchanger together with the flow rate is used to estimate the total heat being taken up by the freon system. Assuming all beam RF power is ultimately absorbed in the freon, loss factors $k$ were calculated and given in Table 1 . These measurements indicate the overall loss factor is low, as expected from impedance measurements on mock-ups [6].

A significant amount of power generated by photocurrents driven by the high voltage power supplies causes some overestimate of the loss factor. On the other hand, there is also some cooling of the body of the separator by tunnel air which is not accounted for in this calculation and would tend to underestimate the loss factor. Limits on the overestimation of loss factor can be determined from measured HV power supply currents and voltages. Photoelectrons striking the positive electrodes ultimately transfer their energy to the freon cooling. The photoelectron kinetic energy at impact will either be equal to the electrode volt-
Table 1: Beam RF loss factors $k$ averaged over one day with typical currents of $190 \mathrm{~mA} /$ beam 36 bunches. Also given is the calculated beam RF power at higher current levels. The average daily power during the measurement in the $45 \mathrm{~W}$ separator was $800 \mathrm{~W}$.

\begin{tabular}{llll}
\hline \hline Name & $\mathrm{k}[\mathrm{V} / \mathrm{pC}]$ & $\begin{array}{l}\text { Power [W] } \\
300 \mathrm{~mA}\end{array}$ & $\begin{array}{l}\text { Power [W] } \\
500 \mathrm{~mA}\end{array}$ \\
\hline $8 \mathrm{E}$ & 0.11 & 1918 & 3196 \\
$45 \mathrm{E}$ & 0.13 & 1837 & 3061 \\
$45 \mathrm{~W}$ & 0.14 & 2439 & 4065 \\
$8 \mathrm{~W}$ & 0.11 & 2281 & 3802 \\
\hline Ave. & 0.124 & 2119 & 3531 \\
\hline \hline
\end{tabular}

age or double it, depending on whether it originates at a grounded surface or on the negative electrode. For the $45 \mathrm{~W}$ separator this works out to a range of between $120 \mathrm{~W}$ and $240 \mathrm{~W}$, amounting to $15-30 \%$ of the total power.

\subsection{Synchrotron Radiation and Photoelectron Current}

Because synchrotron radiation cuts a swath in the horizontal plane, the electrodes for the horizontal separators are made in two pieces with the midplane missing. See Figure 2. A small bridge connects the upper and lower halves and is masked by apertures at the end of the separators, so direct synchrotron radiation cannot strike the electrodes. Nevertheless a strong photoelectron current is observed, so the photoelectrons must be generated by scattered radiation. The scattered SR photons come from two sources: scattered photons from the SR absorbers built into the sep- 


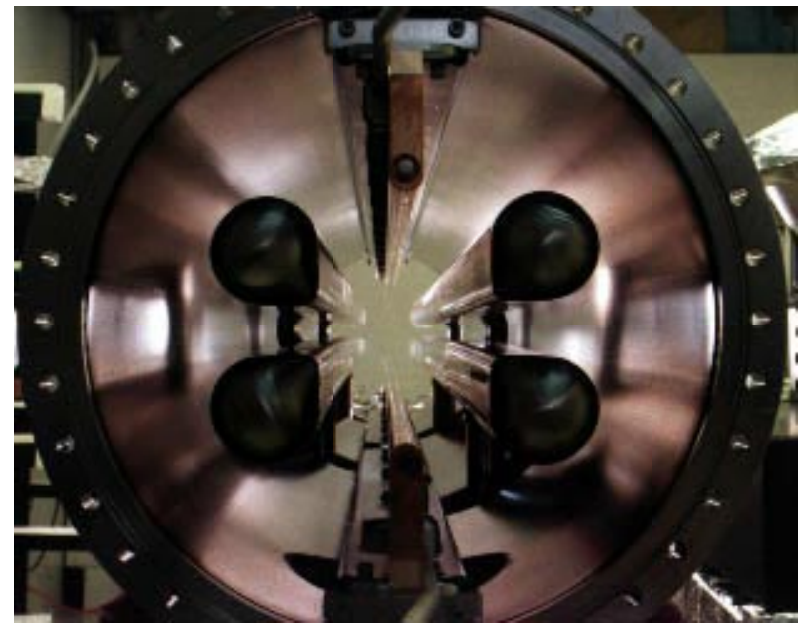

Figure 2: The electrode structure of the CESR separators.

Table 2: Coefficients which determine the high voltage power supply photocurrent from the electron and positron currents in the machine. The definition of the terms is $I_{\text {ps }}=\alpha I_{\text {positron }}+\beta I_{\text {electron }}$

\begin{tabular}{lrrrr}
\hline \hline Separator & $\alpha+$ & $\beta+$ & $\alpha-$ & $\beta-$ \\
\hline $8 \mathrm{E}$ & 0.0018 & 0.0012 & 0.0028 & 0.0006 \\
$45 \mathrm{E}$ & 0.0074 & 0.0059 & 0.0018 & 0.0004 \\
$45 \mathrm{~W}$ & 0.0074 & 0.0007 & 0.0142 & 0.0022 \\
$8 \mathrm{~W}$ & 0.0009 & 0.0024 & 0.0000 & 0.0000 \\
\hline \hline
\end{tabular}

arators, and SR scattered photons from adjacent vacuum chambers. It appears that the latter dominates at CESR.

Intrepretation of power supply current as photocurrent is straightforward. The power supply current is almost independent of voltage above a few $\mathrm{kV}$. It is quite linearly proportional to beam current. In most cases it is much larger the field emission current. The photocurrents depend somewhat on the closed orbit and the photoemission coefficients are not constant in time. However, these effects are at the $50 \%$ level or less. The linearity with beam current is diagnostic and was used to determine coefficients of photocurrent versus beam current given in Table 2. The data was based on one day of high current running. With no beam the current drawn is about $0.03 \mathrm{~mA}$ on each supply.

\subsection{Spark rate}

Low spark rate is critical to efficient operation of the storage ring. In the past high spark rates have been obtained episodically; that is they come in groups. In some cases sparks have been attributed to failing cables or connectors, but in most cases the cause was either a vacuum arc, or an arc on the power supply side of the spark detector whose cause was undetermined. During the first two years of operation the spark rate was much higher, typically 1 spark per day per separator. After new high voltage power supplies were installed the rate dropped dramatically. It is not clear why this occurred but a prime suspect is that control electronics (which were replaced with the new supplies) may have been turning off the supplies unnecessarily. There does not seem to be any general correlation with beam current though occasionally it was observed in the past. At the present time the spark rate is practically neglible. In the past three months of operation there was only one recorded spark that caused significant beam loss.

\subsection{Other Operational Issues}

A few miscellaneous issues are discussed in this section. Not long after the separators were installed, the Clean Air Act was revised to include many new procedures and restriction on freon usage because it is an ozone depleter. We chose to keep Freon 113 as the primary coolant/dielectric fluid, as no other alternate could perform as well in the RF absorbers. It was necessary to rebuild the plumbing systems to make them 'hard sealed' and preserve the existing freon supplies. Since then, freon loss rates have been neglible and the freon has served well. Radiation damage to a HV power supply occurred several times at one location, until it was understood what was happening. Voltage slewing rate when dropping voltage has been of concern. Most power supplies have a high photocurrent drain load on the power supplies and as a result bleed down rapidly. One separator is apparently well shielded from SR and required an external resistor shunting about $1 \mathrm{~mA}$ for good bleed down times.

\section{REFERENCES}

[1] D.L. Rubin, CESR Status and Plans, (PAC95), vol. 1, pp 481f, CBN 95-8 http://www.Ins.cornell.edu/public/CBN

[2] W. Kalbreier, N. Garrel, R. Guinard, R.L. Keizer, K.H. Kissler, Layout, Design, and Construction of the Electrostatic Separator System of the LEP $e^{+} e^{-}$Collider, Proc. EPAC, vol 2, June 1988, or CERN SPS/88-20 (ABT)

[3] T. Shintake, Y. Suetsugu, K. Mori, M. Sato, T. Higo, Design and Construction of Electrostatic Separators for TRISTAN Main Ring, KEK Report 88-17, March 1989 A.

[4] B. Goddard, Separator Performance with Bunch Trains and Pretzel, Proc. 6th LEP Performance Workshop, Chamonix, (1996)

[5] R.M. Littauer, B.D. Mcdaniel, D. Morse and D.H. Rice, "Proposed Multibunch Operation Of Cesr," IN *BATAVIA 1983, PROCEEDINGS, HIGH ENERGY ACCELERATORS*, 161-163. .

[6] J.J. Welch, Z.X. Xu, Low Loss Parameter for New CESR Electrostatic Separators, Proc IEEE Particle Accelerator Conference, May 1991, vol 3., pp $1851 \mathrm{f}$.

[7] J.J.Welch, High Voltage Sparking in a Decommissioned CESR Horizontal Separator, CBN 94-7, http://www.Ins.cornell.edu/public/CBN/1994

[8] Personal communication. 